\title{
Coocurrencia entre ansiedad y autismo. Las hipótesis del error social y de la carga alostática
}

\author{
Isabel Paula-Pérez
}

Introducción. El concepto de comorbilidad en trastornos del neurodesarrollo como el autismo resulta, en ocasiones, ambiguo. La coocurrencia entre ansiedad y autismo es clínicamente significativa; sin embargo, no siempre es fácil diferenciar si se trata de una comorbilidad 'real', donde las dos condiciones comórbidas son fenotípica y etiológicamente idénticas a lo que supondría dicha ansiedad en personas con un desarrollo neurotípico; si se trata de una ansiedad fenotípicamente alterada por los procesos patogénicos de los trastornos del espectro autista, resultando en una variante específica de éstos, o si partimos de una comorbilidad falsa derivada de diagnósticos diferenciales poco exactos.

Desarrollo. El artículo plantea dos hipótesis explicativas de dicha coocurrencia, que se retroalimentan entre sí y que no dejan de ser una reflexión en voz alta partiendo de las evidencias científicas con las que contamos. La primera es la hipótesis del 'error social', y considera que el desajuste en el comportamiento social de las personas con autismo -fruto de alteraciones en los procesos de cognición social-contribuye a exacerbar la ansiedad en el autismo. La segunda hipótesis, la de la carga alostática, defiende que la ansiedad es la respuesta a un estrés crónico, al desgaste o agotamiento que produce la hiperactivación de ciertas estructuras del sistema límbico.

Conclusiones. Las manifestaciones prototípicas de la ansiedad presentes en la persona con autismo no siempre se relacionan con las mismas variables biopsicosociales evidenciadas en personas sin autismo. Las evidencias apuntan a respuestas hiperreactivas de huida o lucha (hipervigilancia) cuando la persona se encuentra fuera de su zona de confort, y apoyan la hipótesis del 'error social' y de la descompensación del mecanismo de alostasis que permite afrontar el estrés.

Palabras clave. Carga alostática. Comorbilidad. Coocurrencia. Trastornos de ansiedad. Trastornos del espectro autista.

\section{Introducción}

Existe una preocupación creciente por evaluar y tratar la coocurrencia de condiciones psiquiátricas en personas con trastornos del espectro autista (TEA), siendo la ansiedad una de las más comunes. En los últimos años, los clínicos han tomado conciencia del número significativo de personas con diagnóstico de TEA que tiene que batallar diariamente con una variedad de síntomas ansiosos, de mayor o menor intensidad. El papel que la sintomatología ansiosa desempeña en la presentación clínica de los TEA ha ido cambiando a lo largo de los años, y sigue siendo fuente de confusión y controversia. No ha sido hasta muy recientemente cuando ha comenzado a recibir la atención que el tema merece a nivel científico, a pesar de las grandes lagunas que todavía persisten.

Kanner [1] sugirió en los años cuarenta que algunos de los síntomas nucleares del autismo -en especial, el deseo de invarianza ambiental y los repertorios de patrones de comportamiento, actividades e intereses restringidos o estereotipados- están impulsados por la ansiedad. Ésta ya está presen- te en algunas descripciones que hizo de sus 11 casos en 1943: 'el comportamiento del niño está gobernado por un deseo obsesivo-ansioso de mantener la igualdad; [...] incluso cambios menores en el entorno pueden inducir a confusión y distrés, y el miedo a un posible cambio puede convertirse en una fuente de ansiedad.'

En 1965, Despert [2] interpretó los comportamientos e intereses restringidos y estereotipados de los niños con autismo como defensas contra la ansiedad abrumadora que experimentan en el mundo social. Unos años después, Wing y Gould [3] pusieron de relieve la heterogeneidad de la manifestación de la ansiedad en el autismo, diferenciando entre el subtipo activo, pero extraño, que tiende a mostrar reacciones emocionales extremas en determinadas situaciones, y el subtipo distante, que se muestra completamente ajeno a los cambios ambientales.

Posteriormente, referentes como Schopler y Mesibov [4] plantearon la resistencia a los cambios en el autismo como una reacción a las dificultades para comprender qué sucede y a un sentimiento de incertidumbre permanente. También los comportamientos restringidos y estereotipados han sido vistos como
Departamento de Métodos de Investigación y Diagnóstico en Educación. Universitat de Barcelona. Barcelona, España.

Correspondencia:

Dra. Isabel Paula Pérez. Universitat de Barcelona. Campus Mundet. Edif. Llevant, 2. piso, desp. 275. Pg. Vall d'Hebron, 171 E-08035 Barcelona.

E-mail:

isabelpaula@ub.edu

Declaración de intereses: La autora manifiesta la inexistencia de conflictos de interés en relación con este artículo.

Aceptado tras revisión externa: 07.01.13.

Cómo citar este artículo: Paula-Pérez I. Coocurrencia entre ansiedad y autismo. Las hipótesis del error social y de la carga alostática. Rev Neurol 2013; 56 (Supl 1): S45-59.

(C) 2013 Revista de Neurología 
consecuencias de la ansiedad, provocados por las limitaciones en la comprensión del entorno. Dicha ansiedad actúa como estrategia de enfrentamiento autocalmante, como papel clave para mantener el miedo y la angustia bajo control [5].

Resulta significativa la aportación de Rescorla [6] en el análisis factorial que realizó con la Child Behavior Checklist [7], un instrumento general de conducta en la infancia que permite la valoración de una amplia gama de psicopatologías. El análisis demostró que los casos más graves de autismo se diferenciaban de los más leves en función de la presencia o ausencia de ansiedad.

Si nos centramos en el Manual diagnóstico y estadístico de los trastornos mentales (DSM) en sus dos últimas versiones, la confusión sobre si los síntomas ansiosos son manifestaciones primarias o secundarias en los TEA está servida. El DSM-III [8] reconoce la presencia de ansiedad inusual e intensa en la descripción del 'autismo infantil'. En él, la ansiedad desempeña un papel importante y se contemplan como criterios diagnósticos síntomas relacionados con la regulación emocional, como: ansiedad excesiva y súbita, reacciones catastróficas a las situaciones de la vida diaria, incapacidad para ser consolado cuando se altera, crisis de angustia y reacciones de rabia inexplicables y labilidad emocional extrema. Sin embargo, en el DSMIV [9] y en su versión revisada [10], todo ese arsenal de síntomas ansiosos se esfuma y se limita a considerar que las personas con autismo pueden exhibir 'una falta de miedo en respuesta a peligros reales y un temor excesivo en respuesta a objetos inofensivos'.

Lo que está fuera de dudas, desde el punto de vista clínico y experimental, es que la prevalencia y gravedad del fenómeno de la ansiedad, con sus diferentes presentaciones -ansiedad social, ansiedad de separación, ansiedad generalizada, trastorno obsesivo compulsivo, fobias específicas...-, desempeña un papel importante y específico en las dificultades de las personas con autismo que excede los niveles registrados en la población general.

Sin embargo, el concepto de comorbilidad en los trastornos del neurodesarrollo, donde las patogénesis no son claras, resulta, en ocasiones, ambiguo. Por ello se hace complicado determinar si la coocurrencia de ansiedad y autismo representa [11]:

- Una comorbilidad 'real', donde la condición comórbida -la ansiedad- es fenotípica y etiológicamente idéntica a lo que supondría dicha ansiedad en personas con un desarrollo neurotípico. En este caso, ¿las manifestaciones prototípicas de ansiedad clínica presentes en la persona con TEA se relacionan con las mismas variables biopsicosociales evidenciadas en personas sin TEA?

- Una ansiedad fenotípicamente alterada por los procesos patogénicos de los TEA, que resulta en una variante específica de los TEA. ¿Se trataría de un aspecto de la diátesis de los TEA, con una posible diferencia etiológica de las personas con TEA que no manifiestan ansiedad? Es decir, si se trata de subtipos únicos de TEA más que de una verdadera comorbilidad, dado que no todas las personas con TEA manifiestan ansiedad.

- Una comorbilidad falsa derivada de diagnósticos diferenciales poco exactos; por ejemplo, confundir sintomatología del trastorno obsesivo compulsivo con los patrones de comportamiento, actividades e intereses restringidos y estereotipados de los TEA [12].

Otro ejemplo es el de la fobia social, que tanto en personas con o sin autismo hace referencia a un temor acusado y persistente en situaciones sociales o actuaciones en público en las que el individuo se ve expuesto a otros que no pertenecen al ámbito familiar o a la posible evaluación por parte de los demás. Los comportamientos de evitación, la anticipación ansiosa o el malestar que aparece en la situación social o actuación en público temida interfieren acusadamente con la rutina normal de la persona, en sus relaciones laborales, académicas o sociales, o bien producen un malestar clínicamente significativo. Teniendo en cuenta que los TEA se definen en parte como una alteración cualitativa de la sociocomunicación, sin clarificar si la evitación social está relacionada con la ansiedad, con la motivación o con ciertas habilidades, el diagnóstico dual exacto se hace muy complicado.

En este artículo se opta por hablar de coocurrencia y no de comorbilidad para hacer referencia a la presencia de ansiedad en las personas con TEA, debido a la falta de evidencias sobre la patogénesis de la ansiedad y del autismo. El último metaanálisis sobre la coocurrencia de la ansiedad con significación clínica en personas con autismo revisó 31 estudios que implicaron a 2.121 personas con autismo menores de 18 años y con las que se evaluó la sintomatología ansiosa que presentaban a través de cuestionarios estandarizados [13]. A lo largo de los estudios analizados, se halló que el $39,6 \%$ de las personas con TEA menores de 18 años presentaba algún trastorno de ansiedad. Los más frecuentes son la fobia social (30\%), seguidos por el trastorno obsesivo compulsivo (17\%), la agorafobia (17\%), la ansiedad generalizada (15\%), la ansiedad de separación (9\%) y las crisis de angustia (2\%). El 10\% res- 
tante correspondía a otros síntomas de ansiedad. Dicha prevalencia resulta coherente con revisiones previas de la bibliografía [14-17].

La investigación genética está todavía lejos de dar una respuesta clara a los interrogantes planteados. Sin embargo, los estudios basados en la psicología experimental y la neurología aportan los primeros indicadores con evidencias significativas para barajar dos hipótesis de trabajo que comiencen a explicar la coocurrencia planteada: la hipótesis del 'error social' y la hipótesis de la 'carga alostática'. Ambas se retroalimentan entre sí, pero no serían las únicas que explican este fenómeno. Todavía hay muchas lagunas en el ámbito de la investigación genética y la neurobiología, explicitadas en el apartado de la prospectiva de la investigación de las conclusiones.

\section{De la condición universal de la ansiedad a la ansiedad clínicamente significativa}

La ansiedad es una condición universal y generalizada a todas las personas, que hunde sus raíces en una emoción tan básica y primaria como es el miedo. No es, pues, esta ansiedad la que preocupa clínicamente hablando. El miedo surge como manifestación saludable del instinto de preservación, que permite a la especie humana sobrevivir y perpetuarse. En el momento en que percibimos una amenaza real que puede comprometer nuestra integridad física o psicológica, nuestro sistema nervioso dispara un mecanismo defensivo de huida o lucha. Eso es el miedo. El miedo responde a un estímulo específico y tiende a tener una duración breve, desapareciendo una vez que la amenaza se ha disipado. 'El miedo es un estado neurofisiológico automático primitivo de alarma que conlleva la valoración cognitiva de una amenaza o peligro inminente para la seguridad física o psíquica de un individuo' [18].

En ocasiones, tales amenazas son malinterpretadas por la persona como reales, cuando verdaderamente la situación más bien pudiera calificarse de neutral o no potencialmente peligrosa. El mecanismo 'miedoso' de huida o lucha se manifiesta en este segundo caso con la misma intensidad y da como resultado una respuesta desadaptativa que, lejos de preservar la integridad física y psicológica de la persona, la deteriora. Ésa es la ansiedad clínicamente preocupante y sobre la que se coloca el foco, la que puede experimentarse en ausencia de una amenaza física directa y persiste mucho más en el tiempo que el miedo. Algunos investigadores la de- nominan 'estado de miedo sostenido' [19]. 'La ansiedad es un sistema complejo de respuesta conductual, fisiológica, afectiva y cognitiva, que se activa al anticipar sucesos o circunstancias que se juzgan como muy aversivas porque se perciben como acontecimientos imprevisibles, incontrolables, que potencialmente podrían amenazar los intereses vitales de un individuo' [18].

Para reconocer dicha ansiedad maladaptativa, referente a un miedo excesivo y clínicamente significativo, han de plantearse cinco interrogantes que marcan la frontera -siempre inestable y difuminada- entre la ansiedad adaptativa y la ansiedad patológica [18]:

- ¿Se basa el miedo o la ansiedad en una asunción falsa o en un razonamiento erróneo relativo al potencial de amenaza o peligro de una determinada situación? Se valora si se perciben los estímulos más amenazadores de lo que realmente son, fruto de cogniciones disfuncionales o distorsiones cognitivas cometidas por errores en el procesamiento de la información.

- ¿Interfiere el miedo o la ansiedad sobre la capacidad de la persona para afrontar las circunstancias aversivas o difíciles? El DSM-IV-TR lo marca como: 'malestar clínicamente significativo o deterioro social, laboral o de otras áreas importantes de la actividad del individuo, o que interfieren de forma notable con su capacidad para llevar a cabo tareas indispensables' [9]. Se produce una inadecuación en la manera en la que se enfrenta la amenaza percibida, dificultando un funcionamiento social eficaz y adaptativo.

- ¿Está presente la ansiedad durante un período prolongado? Esta pregunta remite a la persistencia de la conducta ansiosa en el tiempo más de lo que cabría esperar.

- ¿Experimenta la persona falsas alarmas o crisis de angustia? Es decir, si la ansiedad se manifiesta en ausencia de estímulos objetivamente amenazadores, en ausencia de señales de amenaza o en presencia de 'falsas alarmas'.

- ¿Se activa el miedo o la ansiedad ante una gama relativamente amplia de situaciones que presentan un potencial leve de amenaza? Los detonantes de la ansiedad pueden ser una amplia gama de estímulos y situaciones no amenazantes en población no clínica, lo que nos conduce a considerar la hipótesis de la hiperreactividad a estímulos ambientales.

La respuesta a estos interrogantes ayudará a determinar si la experiencia ansiosa es lo suficientemente distorsionada y generalizada como para requerir 
evaluación adicional, diagnóstico y posible tratamiento. El DSM-IV-TR ofrece una respuesta categorial a los diferentes subtipos específicos de la ansiedad: crisis de angustia (panic attack), agorafobia, trastorno de angustia con y sin agorafobia, agorafobia sin historia de trastorno de angustia, fobia específica, fobia social, trastorno obsesivo compulsivo, trastorno por estrés postraumático, trastorno por estrés agudo, trastorno de ansiedad generalizada, trastorno de ansiedad debido a enfermedad médica, trastorno de ansiedad inducido por sustancias y trastorno de ansiedad no especificado [9]. El borrador del futuro DSM-5 saca de esta clasificación al trastorno obsesivo compulsivo y al trastorno por estrés y trauma, e incorpora los trastornos por ansiedad de separación, que, en la actualidad, se encuentran en 'otros trastornos de la infancia, la niñez y la adolescencia'.

\section{Hipótesis del "error social"}

El cerebro neurotípico analiza, modula, integra, organiza y elabora un juicio en relación con la información entrante, de manera que la persona contextualiza lo que sucede a través de un proceso de inferencia social: extrae el significado de una situación social generando expectativas sobre el comportamiento de los demás y prediciendo lo que harán; prevé qué sucederá; intuye/sabe lo que los otros piensan, sienten, esperan y desean; valora la situación; elabora un juicio social; y toma una decisión. No todas las situaciones sociales son exactamente iguales, y no siempre las reglas y normas son claramente explícitas. Algunas son, verdaderamente, muy sutiles.

Las personas dentro del espectro autista topan con una serie de disfunciones cognitivas en el proceso de integrar la información, analizarla y darle un sentido que obstaculiza el proceso de elaborar un juicio y tomar una decisión para la ejecución de un funcionamiento social efectivo. Esas disfunciones cognitivas se resumen básicamente en:

- Alteración de la pragmática del lenguaje. Déficits para inferir la información que no es explícita, que no está escrita, que es sutil, los factores extralingüísticos que condicionan el lenguaje y que nos habilitan para reconocer las claves y normas sociales que regulan la interacción social: el contexto sociocultural, el tipo de relación con las personas que hablamos, quién está presente o no, la información que compartimos con el interlocutor y la que no, etc.

- Alteraciones en el reconocimiento de emociones propias y ajenas (disleximitia). Dificultades para identificar necesidades señaladas por la emoción, para diferenciar una emoción de otra, no sólo respecto a los propios sentimientos, sino también respecto a las emociones de los demás en sus voces, expresiones faciales, posturas. El término 'dislexitimia' proviene de las raíces griegas dis (alteración), lexis (palabra) y thimos (afecto), es decir, alteración de las palabras para los afectos.

- Déficit en las habilidades mentalistas. Dificultad para atribuir estados mentales y emocionales en los demás; para anticipar comportamientos, intenciones, intuir o saber aquello que los motiva, pasando por alto información social clave para afrontar esa situación; para captar las pistas y claves que rigen el mundo social, por lo general, complejas, cambiantes, muchas veces impredecibles y poco consistentes; para identificar situaciones sociales o personas seguras o no, lo que puede llegar a ponerle en riesgo, etc.

- Disfunción ejecutiva. Dificultad para planificar, elegir, diseñar, crear y mantener la información que es útil para el desarrollo de una tarea; la flexibilidad cognitiva o habilidad para transicionar a un pensamiento o acción diferente en función de los cambios que se dan en cada situación; y dificultades para el establecimiento de metas, prever cosas que no han pasado pero que somos capaces de imaginar y representar en nuestra mente para después plantearnos los objetivos adecuados con la planificación adecuada para cumplirlos.

Finalmente, el cerebro neurotípico ejecuta una respuesta o la inhibe (output) en función de la valoración y juicio que realiza sobre la información entrante. La persona actúa en función de la decisión que ha tomado en la fase previa, y dice, hace, siente o piensa algo. En esta fase, los errores en el procesamiento de la información social se traducen en: malentendidos producto de una interpretación literal del lenguaje; dificultad para comprender metáforas, bromas e ironías; pobres habilidades conversacionales, no respetar los turnos de palabras, no detectar el interés del otro en relación con lo que cuento, si el otro tiene prisa o no, si es adecuado acaparar la conversación y no dejar hablar a la otra persona, si la otra persona es la adecuada a quien dirigir el discurso que está haciendo; no contextualizar lo que se está explicando la interlocutor; no adaptar el contenido de lo que se dice y lo que se hace en función de la especificidad situacional; hablar demasiado o no hacerlo cuando corresponde; decir cosas inapropiadas en el momento equivocado; no entender lo que la gente quiere de ellos, qué han de hacer o decir; no ajustar su comportamiento 
a la intensidad de la emoción; dificultades para desarrollar un proceso ejecutivo, poseer un plan propio, conocer o intuir los planes que tienen los otros en su mente, pudiéndose formar una representación mental de ello y considerar las consecuencias de ambos planes, los propios y los ajenos. El desajuste en el comportamiento social fruto de alteraciones en el funcionamiento de la cognición social es lo que denominamos 'error social'.

En el momento en que la expresión de los síntomas del autismo entra en conflicto con las expectativas y las demandas sociales -o cuando pueden provocar el rechazo de los demás-, la persona se siente confundida y agobiada en contextos sociales, algunos incluso 'se ponen la máscara' y aprenden a disimular sus síntomas y a fingir 'que son normales', evitan las situaciones problemáticas, se exacerban las dificultades interpersonales, aumentando el sentimiento de aislamiento y soledad. Las experiencias sociales negativas y los fracasos reiterados en sus relaciones interpersonales pueden contribuir a la aparición de ansiedad $[20,21]$. Sin embargo, es importante matizar que en el estudio de White y Roberson [22] se halló que los jóvenes con TEA que informaron de mayores niveles de ansiedad también informaron de una mayor soledad social, pero no emocional. Es posible que deseen un mayor contacto social con iguales, pero que sus 'necesidades' de establecer lazos afectivos con otras personas se vean cubiertas con otras personas en sus vidas, como puedan ser sus familiares. Otra hipótesis sería que el hecho de no tener una necesidad tan grande de establecer lazos emocionales les conduzca a que los sentimientos de soledad emocional no sean tan acusados. Estos dados son consistentes con los hallados por Bauminger et al [23].

Es significativa la relación entre altos niveles de ansiedad y mayor gravedad de los síntomas de los TEA, como, por ejemplo, los patrones repetitivos y estereotipados de comportamiento [24]. ¿Son los patrones repetitivos y estereotipados de comportamiento efecto de la ansiedad o mecanismo de afrontamiento ante ésta? El comportamiento repetitivo que manifiestan las personas con TEA ha sido asociado frecuentemente con la ansiedad. Las estereotipias, la rigidez e inflexibilidad cognitiva y conductual, la insistencia en la invariancia ambiental, los rituales y rutinas, las ecolalias inmediatas o diferidas se acentúan en situaciones de ansiedad.

Los altos niveles de ansiedad en personas con autismo también se han asociado a mayor presencia de comportamientos repetitivos $[25,26]$. A mayor nivel de ansiedad, mayor presentación de comportamientos repetitivos e intereses restringidos [24], o, dicho de otra manera, la manifestación de comportamientos repetitivos e intereses restringidos suele tener una función de reducción de la ansiedad y, por tanto, ser consecuencia de la ansiedad $[27,28]$.

Rodgers et al [26] analizaron la relación entre ansiedad y comportamientos repetitivos en dos grupos de niños con autismo: el primero con altos niveles de ansiedad y el segundo con bajos. Los resultados arrojaron evidencias en cuanto a que aquellos niños con mayores niveles de ansiedad mostraban también más comportamientos repetitivos que los que mostraban niveles de ansiedad más bajos. Del grupo con mayores niveles de ansiedad, ésta se asoció a un mayor deseo de invariancia ambiental y no tanto a estereotipas sensoriales motoras. En cambio, en el grupo con menor nivel de ansiedad, ésta sí se asoció de manera significativa con estereotipas sensoriales motoras. Estos resultados concuerdan con los de Spiker et at [28] y Joosten et al [29].

Otra consecuencia del 'error social' que conviene no ignorar y que la investigación aborda poco es la presencia de hostilidad y otras formas de agresión ligadas a la experiencia de ansiedad en personas con TEA. En estudios realizados con personas neurotípicas, la ansiedad social se asocia con frecuencia a sentimientos de ira o enojo hacia los demás. La persona considera que está siendo relegada al ostracismo, se siente excluida en su entorno social o es criticada y juzgada, lo que le hace adoptar un punto de vista poco favorable en relación con los demás y experimentar esos sentimientos de hostilidad [30,31], y, en ocasiones, entrar en un estado de hipervigilancia de potenciales situaciones hostiles de los demás hacia él/ella. Esta hipervigilancia a fuentes potenciales de evaluación negativa predispone a la persona a percibir hostilidad en los demás y a poner en juego sentimientos negativos hacia ellos [32]. Así pues, en los adolescentes neurotípicos existen evidencias de que las atribuciones hostiles actúan de mediadoras entre el rechazo de los iguales y la agresión hacia los demás [33].

El comportamiento hostil y agresivo en personas con TEA ha empezado a estudiarse recientemente con datos experimentales $[34,35]$. El estudio de White et al [36] analiza si los síntomas de ansiedad actúan como mediadores en la relación entre los TEA de alto funcionamiento y los sentimientos de hostilidad en jóvenes adultos. Se halló que la ansiedad mediaba parcialmente entre los síntomas autistas y los autoinformes sobre hostilidad. Hostilidad que se traduce en animadversión caracterizada por una serie de atributos, relativamente estables, de actitudes negativas y resentimiento hacia los demás. Se podría decir que la hostilidad es el resultado de expe- 
riencias interpersonales aversivas. Algunos de los factores que pueden contribuir a las dificultades para manejar el enfado o la ira que conducen a comportamientos agresivos pueden ser: la disfunción ejecutiva relacionada con la dificultad para inhibir respuestas prepotentes [37], la reacción impulsiva a señales emocionales [38], y la alteración en el reconocimiento y la regulación emocional [39].

El papel que desempeña la conciencia de las propias limitaciones y del rechazo social puede ser un dato relevante que hay que tener en cuenta. Las personas con autismo de alto funcionamiento muestran más miedo a ser juzgados por otros o a ser humillados por sus propias acciones. Es lógico conjeturar que, si existe una mayor conciencia (insight) de su alteración sociocomunicativa, pueden experimentar más ansiedad al aumentar expectativas cognitivas negativas relacionadas con el fracaso social. Es un dato claro que las personas con autismo reciben menos aprobación por parte de los iguales y sufren un mayor nivel de rechazo o exclusión social [40]. Esta toma de conciencia contribuye al desarrollo de problemas de ansiedad secundarios y a un aumento del miedo a la evaluación negativa de los iguales [24,41].

La ansiedad en estos casos aparece, pues, como fenómeno secundario que resulta de la autoconciencia de la persona de sus propias limitaciones a la hora de afrontar situaciones en las que ha de poner en juego comportamientos y juicios sociales apropiados a su edad, lo que les provoca miedo a sentirse juzgados o vergüenza. Ello limita el número de interacciones sociales, priva a la persona de nuevas oportunidades para relacionarse -lo que les permitiría aprender nuevas y más refinadas habilidades sociales-, y se inicia un círculo vicioso de evitación, que aumenta los niveles de ansiedad y la conciencia de un funcionamiento social limitado. Por ejemplo, la fobia social se genera por la preexistencia de experiencias negativas con los iguales, ya que son penalizados por los otros a través de las burlas, incluso llegando a ser victimizados -bullying o acoso moral-, con prevalencias del 6-46\% [42]. Esas experiencias de victimización pueden configurarse en las precursoras de una fobia social creciente.

No obstante, el grado de desacuerdo entre las medidas de autoinformes de los jóvenes con TEA y las que ofrecen familiares y profesionales es muy evidente $[43,44]$. La persona con TEA ofrece prevalencias más bajas de ansiedad en comparación con la información aportada por los clínicos y los padres, que ven la necesidad de que reciban tratamiento. Cuando la persona con TEA autoinforma de sus síntomas, la dificultad para identificar emo- ciones y expresar problemas pueden inhibir aspectos importantes de la valoración de la magnitud de sus dificultades y la habilidad para expresar detalladamente la intensidad de la emoción. Manifiestan mayores problemas para responder a los autoinformes sobre sus síntomas ansiosos por su menor capacidad para el pensamiento abstracto o para comunicarse efectivamente a través del habla, de la expresión facial, y, en ocasiones, para tomar conciencia de los estados internos o la motivación para informar sobre sus síntomas. Por ello, es necesaria más investigación en relación con lo apropiado o no de utilizar autoinformes para evaluar la ansiedad en personas con TEA, por su capacidad para identificar y expresar sus estados internos [20]. De hecho, los autoinformes sobre posible sintomatología ansiosa en jóvenes con TEA son menos fiables que los utilizados con jóvenes sin TEA, a la vista de la comparación de los resultados con los datos obtenidos de las entrevistas con los padres [45].

En los casos de TEA de la parte baja del espectro, los autoinformes pueden ser poco válidos o complicados para aquellos que tengan limitaciones cognitivas significativas. Existe una divergencia entre los estudios que afirman que los autoinformes sobre ansiedad en personas con TEA de alto funcionamiento correlacionan con los informes de los padres [46] y los estudios que afirman que los informes de los padres son mucho más precisos [22,47]. Por ello, sería recomendable una combinación de instrumentos de evaluación como cuestionarios, entrevistas y observaciones del comportamiento, a través de múltiples fuentes de información, como familias, profesionales y personas cercanas. Es importante contar con la información que ofrecen los padres o la familia directa que convive con la persona con autismo, aunque exista la limitación de que ellos no tienen acceso a las experiencias internalizantes de sus hijos, al tratarse de síntomas menos visibles $[48,49]$.

A modo de apunte, hay que comentar que la investigación ha hallado diferencias significativas entre la percepción de ansiedad de los padres en sus hijos a la del profesorado. El profesorado tiende a puntuar más alto la sintomatología ansiosa que los padres [50,51], quizás porque en las escuelas las demandas de todo tipo son más altas y exigentes que en el entorno familiar.

\section{Hipótesis neurobiológica de la carga alostática}

Las reacciones instintivas a situaciones amenazantes generadoras de ansiedad y sus consiguientes respuestas regulatorias responden a un diálogo entre 
procesos de bottom-up y de top-down. Pongamos un ejemplo: vamos por la calle y nos encontramos de frente con un bulldog. Nuestra respuesta inicial es de alerta (proceso de bottom-up), pero la respuesta de afrontamiento a la amenaza aparece mediada por el hecho de observar que no supone un peligro inmediato, puesto que está atado con correa (control top-down). El contexto es un determinante crítico, puesto que si nos encontráramos con el bulldog solo, sin correa ni amo que lo controle, posiblemente iniciaríamos una respuesta de bloqueo seguida por una respuesta de huida o lucha (flight or fight). Precisamente, la interacción entre los procesos de bottom-up y top-down es la que determina la adaptación del comportamiento en cada situación específica y la capacidad para regular adecuadamente nuestras emociones en la vida cotidiana. Así pues, una alteración en la interacción de estos procesos puede desencadenar síntomas de ansiedad más o menos graves, desde la ansiedad 'normal' adaptativa a la ansiedad patológica desadaptativa.

Esas estructuras neurológicas esenciales para el diálogo-interacción entre los procesos bottom-up y top-down son la amígdala, la corteza prefrontal medial y el hipotálamo, que permiten la activación adaptativa ante amenazas reales y posibilitan la inhibición y control ante situaciones de seguridad. Dicho sustrato neuroanatómico se configura como un neurocircuito, no como estructuras separadas, ya que, cuanto mejor se acoplan las diferentes estructuras, mejor predicción podemos hacer del comportamiento en relación con la ansiedad [52]. Estudios de neuroimagen con humanos apuntan a que el circuito implicado en el aprendizaje, el mantenimiento y la extinción del miedo condicionado está sistemáticamente desregulado en personas con rasgos ansiosos o con trastornos de ansiedad clínicamente delimitados [53-56].

Las teorías neurobiológicas de la ansiedad han subrayado el papel clave de la amígdala en la generación y en la experiencia del miedo que conduce a la ansiedad $[57,58]$-filogenéticamente, una de las estructuras cerebrales más arcaicas diseñada para detectar las amenazas del entorno y modelar la respuesta subsecuente de huida o lucha para asegurar la supervivencia-. La amígdala, en conexión con otras regiones cerebrales, sostiene diferentes funciones, entre ellas, la más poderosa para este artículo, el reconocimiento instantáneo y la evaluación de los estímulos emocionalmente relevantes, la generación posterior de los estados afectivos y la regulación autonómica de la respuesta [59]. Una actividad elevada de la amígdala, investigada a través de estudios de neuroimagen funcional, se traduce en ansiedad elevada [60,61], así como en una elevada expresión de afecto negativo durante la adquisición del miedo $[62,63]$. La ansiedad no solamente se asocia a una alta activación de la amígdala en relación con estímulos amenazantes, sino también con estímulos no amenazantes como caras neutras, de manera que la actividad de la amígdala puede traducir altos niveles de ansiedad aun en ausencia de amenazas evidentes [64].

Por su parte, el hipocampo, independientemente de su papel en la memoria, desempeña una función importante en la modulación del contexto del miedo, de manera que éste se adquiere en determinados contextos y guía la expresión del miedo en función de éstos [65]. Lo hace a través de conexiones inhibitorias con la amígdala y otras estructuras implicadas en la percepción de la emoción, permitiendo la producción de un comportamiento afectivo contextualmente apropiado [59]. Además de su papel en el procesamiento emocional y en la memoria [66], el hipocampo también interactúa recíprocamente con la amígdala para codificar los recuerdos emocionales [67]. La atrofia del hipocampo en pacientes clínicamente ansiosos sugiere que la modulación contextual del miedo también puede estar alterada en la ansiedad, de manera que la persona generaliza el miedo a estímulos similares [68].

Para completar el neurocircuito, la corteza prefrontal medial regula y controla el output de la amígdala y la conducta que se sucede, convirtiéndose en una zona de asociación para los procesos de aprendizaje del miedo condicionado, la extinción del miedo, la regulación emocional y la interpretación de expresiones faciales ambiguas, entre otros. Personas con temperamento ansioso manifiestan un acoplamiento débil de la amígdala y la corteza prefrontal medial durante tareas de apareamiento de caras con gesto de enfado y de miedo [69]. También la integridad estructural de las vías amígdala-corteza prefrontal ventromedial correlaciona negativamente con los rasgos ansiosos, de manera que la alteración de la función inhibitoria contribuye a una alta reactividad y a la desregulación emocional en la ansiedad [52,70]. La corteza prefrontal medial cuenta, además, con proyecciones al tronco cerebral y al hipotálamo, que modulan la expresión del miedo, y con proyecciones al estriado ventral, que media en la manifestación de estrategias de enfrentamiento al miedo [58].

Dadas las dificultades de las personas con TEA en el procesamiento y la respuesta a las señales socioemocionales, se sugiere que su base neurobiológica incluye anomalías en el desarrollo y función de las estructuras límbicas, que implican de manera 
especial a la amígdala y al hipocampo [71-75]. Específicamente, se ha hallado un patrón de crecimiento anómalo y disfuncionalidad de la amígdala y el hipocampo en el autismo. Ha resultado decepcionante no hallar investigaciones relevantes sobre posibles alteraciones estructurales y funcionales de la corteza prefrontal medial y el neurocircuito que lo conecta con otras estructuras límbicas, específicamente en personas con autismo. Prácticamente, la totalidad de los estudios con personas con TEA se limitan al estudio de la medición volumétrica y la hiperactivación de la amígdala y el hipocampo, por lo que hemos de tener presente la laguna científica al respecto.

Bauman y Kemper observaron por primera vez en 1985 signos de neuropatología en la amígdala del cerebro de personas autistas post mortem [76]. Estos resultados, además de los datos de neuroimagen funcional, condujeron a Baron-Cohen et al [73] a proponer que la disfunción de la amígdala puede ser responsable, en parte, del deterioro del funcionamiento social en el autismo, rasgo distintivo de esta condición. Posteriormente, se sugirió que la amígdala tenía un mayor volumen en niños con autismo de 36-56 meses de vida [77], y a partir de aquí ya han sido múltiples los datos empíricos que confirman el aumento bilateral de la amígdala -alrededor de un $15 \%$ más grande- en niños con autismo de entre 2 y 4 años en comparación con los grupos control $[78,79]$.

En un estudio prospectivo para medir el volumen de la amígdala en bebés con riesgo de padecer autismo, se midió el volumen de la amígdala a los 2,5 años y se volvió a medir a los 4 años [79]. Se hallaron evidencias de un crecimiento de la amígdala antes de los 3 años de vida en los niños con autismo, justo cuando los síntomas clínicos comienzan a hacerse evidentes. Los resultados son consistentes con los estudios de Mosconi et al [78] y de Sparks et al [77] en cuanto a que la amígdala era desproporcionadamente grande en relación con el volumen cerebral total a los 3 años de edad. En los bebés que recibieron el diagnóstico de autismo con posterioridad, el grado de aumento de la amígdala a los 3 años de edad estaba asociado con la gravedad de las alteraciones de la socialización y la comunicación.

En estudios con jóvenes de entre 7 y 18 años, separados en grupos de edad categóricas -niños y adolescentes-, el agrandamiento de la amígdala era específico de los niños con autismo de entre 7 y 12 años, tanto de bajo como de alto funcionamiento, a pesar de que se halló una mayor tendencia al aumento de la amígdala derecha en aquéllos con síndrome de Asperger. Sin embargo, no se hallaron diferencias significativas en el volumen de la amígdala en los adolescentes de 12,5-18,5 años de edad con síndrome de Asperger y los grupos control [79]. Nuevamente se confirma que las diferencias en el volumen de la amígdala y el hipocampo de las personas con TEA y controles varían según la edad y el subtipo diagnóstico.

Groen et al [80] aportaron evidencias de las propiedades volumétricas del hipocampo y la amígdala en personas con autismo de entre 12 y 18 años sin retraso mental. Aunque no se hallaron diferencias en el volumen total del cerebro entre los dos grupos, si se encontró un mayor volumen estadísticamente significativo de la amígdala derecha y del hipocampo izquierdo, lo cual concuerda con estudios previos [77,81-83].

El único estudio en vivo sobre las diferencias relacionadas con la edad en el volumen de la amígdala y el hipocampo en individuos con TEA desde la infancia hasta la edad adulta con un enfoque continuo es el de Clodagh et al [84], quienes compararon las diferencias relacionadas con la edad en el volumen de la amígdala y el hipocampo en grupos control con desarrollo normal y personas con TEA físicamente sanas (por ejemplo, sin historia de epilepsia) con edades comprendidas entre los 12 y 47 años. El estudio concluyó que las personas con síndrome de Asperger tienen un volumen significativamente mayor tanto de la amígdala izquierda como de la derecha, en concordancia con los resultados de estudios anteriores [77-79,83,85], en contraposición a estudios que no hallaron diferencias en el volumen de la amígdala [86-88], y estudios que hallaron un volumen menor [89-91]. La inconsistencia de los resultados se puede deber a que algunas de estas investigaciones -excepto las de Schumann et al [83], Groen et al [80] y Clodagh et al [84] - incluían grupos relativamente pequeños y clínicamente heterogéneos, que diferían en edad, coeficiente intelectual, criterios de exclusión (por ejemplo, presencia o ausencia de la epilepsia) y métodos de medición [92].

La hipótesis de que las personas con autismo que muestran un crecimiento temprano del cerebro y mayores alteraciones del comportamiento pueden mostrar un tamaño menor de la amígdala en la vida adulta necesita ser validada en estudios longitudinales sobre el crecimiento del cerebro a través del desarrollo de las personas con autismo. Dada la dificultad para evaluar los procesos metabólicos y celulares del cerebro con técnicas no invasivas y la escasez de datos post mortem disponibles de personas jóvenes con autismo, no disponemos de información sobre la base celular que justifique el crecimiento precoz de la amígdala. Podría deberse a un creci- 
miento dendrítico y a una sinaptogénesis más rápidos o a un proceso anormal, como la proliferación glial como parte de un proceso inflamatorio. Pero todo son hipótesis que hay que corroborar. Las evidencias microscópicas de los estudios de neuropatología muestran un aumento de la densidad de las células neuronales, neuronas anormalmente pequeñas, y una disminución del número de neuronas en la amígdala [76,93-95], así como una reducción de las ramificaciones dendríticas del hipocampo [96].

Puesto que la amígdala tiene un papel clave en la regulación socioemocional, su funcionamiento atípico en el autismo predice alteraciones en las habilidades sociocomunicativas [78,79], problemas para establecer contacto visual $[75,89,97]$, dificultades para el reconocimiento facial [98-100] y de las emociones $[92,101,102]$. Pero, para el tema que nos ocupa, es importante destacar su función en el reconocimiento del miedo y el enfrentamiento a la ansiedad $[72,103]$. De hecho, en el autismo, la amígdala sufre anormalidades estructurales y funcionales muy similares a las observadas en casos de ansiedad pediátrica y, posiblemente, también en niños que no han desarrollado ansiedad, pero que tienen un comportamiento desinhibido, tanto por lo que hace referencia a un mayor volumen de la amígdala [104], como por lo que hace referencia a una hiperactivación de ésta [105-107]. La constatación de que la amígdala está hiperactivada en personas con autismo en respuesta a estímulos sociales explica la manera inadecuada en que las personas con TEA miran a la cara y se relacionan $[100,108]$, y es coherente con los datos expuestos en puntos anteriores sobre la percepción de las interacciones sociales como amenazantes y, por lo tanto, susceptibles de ser evitadas para aliviar la angustia que se desencadena.

Una de las hipótesis barajadas que justifica el aumento volumétrico de la amígdala apuesta por un proceso de estrés crónico en el autismo, posiblemente provocado por una hiperexcitación de esta estructura, que da lugar a su hipertrofia inicial [80]. Las personas con TEA tienen un riesgo significativamente mayor de sufrir estrés, que afecta al desarrollo de la amígdala [109]. Dado que el hipocampo tiene un importante efecto regulador sobre la actividad de la amígdala a través de una densa red de conexiones recíprocas, es plausible que el hipocampo también aumente en respuesta a la mayor actividad de la amígdala. En el caso del autismo, el agrandamiento de la amígdala y del hipocampo sería un efecto del esfuerzo de adaptación que realiza la persona con autismo, traducido en una mayor actividad de estas estructuras durante la infancia y la adolescencia. La vida cotidiana, con sus demandas sociales y situaciones constantemente cambiantes, provoca un estrés grave en niños con autismo [110,111].

Otro dato que avala esta hipótesis es el hecho de que el aumento de volumen paralelo del hipocampo y la amígdala durante la infancia y la adolescencia en el autismo perdura más que, por ejemplo, las anomalías volumétricas relacionadas durante un primer episodio depresivo en el que únicamente se ha hallado un agrandamiento de la amígdala [112].

Pero ¿cómo debemos interpretar los datos que revelan una normalización de las medidas volumétricas en la amígdala y el hipotálamo en la adolescencia y la edad adulta, produciéndose incluso una pérdida relativa de volumen? [80]. ¿Forma parte del proceso de evolución del trastorno? ¿Es una repercusión de las primeras etapas de desarrollo anormal? Si la hipertrofia temprana de la amígdala en el autismo conduce a la ansiedad anormal con la activación concomitante de los mecanismos de estrés, la disminución del tamaño de la amígdala podría ser un resultado de la carga alostática y de los procesos de retroalimentación degenerativos, como se observa en la depresión crónica [113]. La alostasis es un mecanismo de protección que moviliza al sistema nervioso central, al sistema endocrino y al inmunitario, y los prepara para afrontar el estrés de una determinada situación. Si la alostasis es ineficaz, inadecuada o el agente que la provoca se prolonga por mucho tiempo, la persona tiene problemas para lograr una buena adaptación, produciéndose la carga alostática. Así pues, la carga alostática es una respuesta continua de estrés desregulada. Este desgaste o agotamiento, que se produce por una hiperactividad del sistema de alostasis, a largo plazo puede causar patologías, tanto orgánicas como psíquicas.

Los estudios con animales han demostrado cómo el estrés provoca inicialmente la hipertrofia de la amígdala [114], pero la desregulación crónica de la respuesta al estrés -la carga alostática- puede conducir a una disminución del volumen de la amígdala y el hipocampo en la edad adulta. Esto ha sido apoyado por estudios sobre la depresión mayor recurrente, una enfermedad que se asocia con el estrés crónico, en la que la disminución del volumen de la amígdala y del hipocampo se da en fases tardías de la enfermedad $[115,116]$. Otros estudios realizados con animales han demostrado que el estrés crónico repetido evoca cambios excitotóxicos en el hipocampo, lo que causa una degeneración de éste [117].

Así pues, las causas que pueden provocar las sutiles diferencias en la morfometría y el desarrollo de la amígdala y el hipocampo no están del todo clarificadas, pero parece ser que se deben a una compleja interacción genética-ambiente. Aunque la inves- 
tigación genética es todavía muy insuficiente, un soporte preliminar indirecto para esta supuesta influencia lo proporcionan las evidencias en las diferencias de volumen de la amígdala y el hipocampo en los familiares de las personas con TEA $[81,89]$.

\section{Conclusiones}

La coocurrencia entre ansiedad y autismo pone en cuestionamiento el concepto de comorbilidad entre ambas manifestaciones clínicas. Cuando hablamos de comorbilidad real entre ansiedad y autismo, estamos confirmando que dicha ansiedad es fenotípica y etiológicamente idéntica a lo que supondría dicha ansiedad en personas con un desarrollo neurotípico. Verdaderamente, ¿las manifestaciones prototípicas de ansiedad presentes en la persona con TEA se relacionan con las mismas variables biopsicosociales evidenciadas en personas sin TEA? Parece ser que no siempre es así.

La ansiedad en personas sin autismo está relacionada con una serie de variables, como, por ejemplo, relaciones sociales pobres o baja autoestima, lo que deteriora el funcionamiento social de la persona en comparación con población neurotípica sin dichos trastornos. Son menos asertivos, inician menos interacciones sociales, muestran menor comportamiento prosocial, mayor aislamiento social, menor calidad en las interacciones, más dificultades para generar conversaciones, tienen menos amigos, son menos aceptados por los iguales, padecen más victimización o se sienten más solos $[118,119]$, de manera que es evidente que una disminución de la sintomatología ansiosa revertirá en una mejora de la conducta social [120,121]. Dicha ansiedad desvía la atención de los estímulos relevantes en pro de estímulos y cogniciones amenazantes que provocan un deterioro en el proceso de resolución de problemas.

En cambio, en las personas con TEA, la ansiedad se relaciona generalmente con:

- Demandas de los demás, que no les permiten desarrollar sus rutinas o intereses restringidos.

- Dificultades para comprender la perspectiva de los demás.

- Burlas y rechazo que reciben por parte de los demás (bullying).

- Alteraciones de la integración sensorial: hiper o hiporreactividad a los estímulos sensoriales o inusual interés en aspectos sensoriales del entorno, entre otros.

Las evidencias apuntan a que las personas con autismo suelen tener una respuesta hiperreactiva en situaciones en que se sienten fuera de su zona de confort. Se muestran hipervigilantes, nerviosos, incapaces de relajarse, molestos, inquietos, y ello puede desencadenar una respuesta ansiosa excesiva. Prácticamente, se podría decir que la ansiedad es para muchos de ellos un estado natural, porque forma parte de su 'maquillaje neurológico'. La constante necesidad de hipervigilancia crea un nivel de ansiedad natural como medio primario de autopreservación. La ansiedad se convierte simplemente en una reacción instintiva y no en un comportamiento aprendido. Lamentablemente, solemos considerar estas reacciones como alteraciones del comportamiento, desafiantes o desobedientes, cuando, en realidad, se trata de una conducta adaptativa instintiva de respuesta a estresores o desencadenantes de la angustia.

Si la investigación clínica apunta a una alta prevalencia de diagnósticos duales entre trastornos de ansiedad y otros trastornos del eje I a lo largo de la vida de un individuo, de manera que la presencia de ansiedad clínicamente significativa predispone a padecer otros trastornos adicionales, ¿no deberíamos abrazar un paradigma dimensional de la ansiedad en lugar del tradicional categorial? En un estudio con 1.127 pacientes, hallaron que dos tercios de los que padecían un trastorno de ansiedad presentaban simultáneamente otro trastorno del eje I, y más de tres cuartos de los pacientes estudiados habían padecido otro trastorno comórbido a lo largo de su vida [122]. Estos datos confirman la coocurrencia diagnóstica de los trastornos de ansiedad como regla y no como excepción, de manera que predispone a la persona que la padece a manifestar uno o más trastornos adicionales.

Para comprender por qué las personas con TEA manifiestan ansiedad en situaciones cotidianas aparentemente inocuas, es necesario comprender cómo perciben el mundo. Por lo general, las rutinas y los rituales gobiernan sus actividades de la vida diaria, por lo que les ayuda disponer de patrones de situaciones que 'guionicen' su vida. En una sociedad en la que precisamente todo es impredecible y está en constante cambio - a menudo sin lógica alguna tras esos cambios-, es normal que las personas con autismo se sientan confundidas, perdidas y sobrepasadas en muchas ocasiones. El mundo social es imprevisible y caótico: a veces lo que se dice no se cumple, o no es verdad, muchas preguntas no tienen respuestas claras, los planes pueden cambiar, existe el engaño, los malentendidos, la imprevisibilidad. Ingredientes complicados para personas que lo que más precisan es lógica, planificación, previsibilidad, invarianza situacional. En ocasiones, algo 
tan sencillo para nosotros como la transición de una actividad o situación a otra puede suponer un obstáculo para la persona con TEA. Estoy leyendo el periódico y suena el teléfono. Dejo de leer y lo atiendo. Cambiar de foco de atención en su caso puede suponer un problema y, cuando se sienten presionados a hacerlo, se desencadenan los síntomas de la ansiedad.

Si a ello añadimos las alteraciones de la integración sensorial, que convierte el entorno en demasiado ruidoso, demasiado luminoso, demasiado sobreestimulante, las posibilidades de sentirse amenazadoramente estresados son muy altas, aunque esas situaciones sean objetivamente neutrales para las personas neurotípicas.

Debora Lipsky [123], una mujer de 45 años con síndrome de Asperger, describió que la técnica de referncia de toda persona con TEA es el scripting ('necesitamos cada segundo de cada minuto de cada hora guionizado'), la necesidad primaria de sentirse seguros y poder predecir lo que va a suceder en el entorno, dada la gran ansiedad que provoca lo impredecible. Salirse del guion o no disponer de él, la falta de estructura en el día a día, los conduce a la pérdida del control y a la angustia. Sin embargo, es muy complicado tener todo absolutamente 'guionizado', por lo que puede ser necesario un espacio para ayudar a la persona a responder a los '¿qué pasa si...?', ofreciéndoles guiones alternativos a situaciones que pueden desarrollarse de maneras diferentes. La espontaneidad suele ser una mal aliada para personas con autismo. La rutina y los rituales, la mejor de las compañías.

Se podría decir que la persona con autismo vive en un mundo de absolutos, sin grises, donde las cuestiones que surgen únicamente pueden resolverse de manera dicotómica. Existe una razón para todo y todo tiene una razón. Por ello, por la imposibilidad de ofrecerles ese mundo de seguridad, el mundo social neurotípico resulta muy poco 'amigable'. Por lo general, aclarar una situación comunicativa que no han comprendido, manipular un estresor ambiental o sensorial, o clarificar una demanda o pregunta puede ser suficiente para, momentáneamente, rebajar los niveles de ansiedad. Éstas son las estrategias preventivas que permitirán minimizar futuros episodios ansiosos. Así pues, el tratamiento de las crisis ha de ser proactivo y no reactivo.

La frontera entre lo que supone una comorbilidad real o una ansiedad propia de determinados procesos patogénicos en el autismo puede ser confusa. ¿Hasta dónde podemos hablar de fobia social o de ansiedad de separación, entre otros trastornos de ansiedad, o un efecto del 'error social' en las per- sonas con TEA? El hecho de que la prevalencia de la ansiedad clínicamente significativa sea muy alta en población con autismo, pero no universal para todas las personas con el diagnóstico, plantea la cuestión de si no estaremos hablando de subtipos de TEA más que de una verdadera comorbilidad.

La prospectiva de la investigación en neurobiología debería apuntar hacia:

- La clarificación del desarrollo de la amígdala y el hipocampo durante toda la vida a través de estudios longitudinales, y la determinación de si difiere entre los subtipos de diagnóstico de TEA.

- La profundización, con estudios combinados de neuroimagen funcional y estructural, de hasta qué punto la ampliación paralela de la amígdala y el hipocampo refleja la adaptación del cerebro de la persona con autismo al desbordamiento continuo de experiencias emocionales a las que tiene que enfrentarse a lo largo del ciclo vital, lo que podría explicar la manifestación de altos niveles de ansiedad, el comportamiento agitado en respuesta a cambios repentinos y la sobreexcitación (overarousal) en respuesta a determinados estímulos.

- El establecimiento de si estas anomalías de la amígdala y el hipocampo están causadas por una mala regulación primaria dentro de estas estructuras límbicas o la deficiente regulación top-down de las áreas prefrontales del cerebro.

En relación con la evaluación de los trastornos de ansiedad en población con TEA, la revisión de los estudios revela una limitación respecto de los instrumentos de evaluación utilizados. Éstos son los mismos que para la población general, sin que existan evidencias de la validez y fiabilidad de dichos instrumentos diagnósticos para las personas con TEA. La pregunta clave es: $¿$ son los instrumentos de evaluación y diagnóstico de la ansiedad diseñados para la población neurotípica fiables y válidos para las personas con autismo? Parece ser que la validez de las entrevistas diagnósticas para jóvenes y adultos con autismo no está muy clara. Se recomienda, pues, explorar los mecanismos subyacentes que puedan explicar el desacuerdo en las puntuaciones entre las personas con TEA y sus padres; analizar los posibles síntomas internalizantes en los TEA de alto funcionamiento, debido a que son más vulnerables por su mayor conciencia social y su interés por las relaciones sociales en comparación con personas con autismo de la parte baja del espectro; y diseñar métodos de evaluación e instrumentos que aborden las características distintivas y únicas de la ansiedad en personas con TEA. 
Por último, en cuanto a la prospectiva de la investigación, sería interesante evaluar la presencia de sintomatología ansiosa a través de datos postratamiento, de manera que, cuando aplicamos la intervención cognitivo conductual específica para los trastornos de ansiedad, los síntomas de la ansiedad disminuyen en intensidad o remiten, mientras que se mantienen los síntomas nucleares del autismo [124-126]. De hecho, en el estudio de Wood et al [127] sobre terapia cognitivoconductual para trastornos de ansiedad coocurrentes en personas con autismo, se observó que, a medida que remitían los síntomas de la ansiedad durante el tratamiento, los padres informaban de una mejora de la sintomatología autista.

\section{Bibliografía}

1. Kanner L. Autistic disturbances of affective contact. Nerv Child 1943; 2: 217-50.

2. Despert JL. The emotionally disturbed child. New York: Brunner; 1965.

3. Wing L, Gould J. Severe impairments of social interaction and associated abnormalities in children: epidemiology and classification. J Autism Dev Disord 1979; 9: 11-29.

4. Schopler E, Mesibov, eds. Behavioural issues in autism. New York: Plenum; 1994.

5. Howlin P. Children with autism and Asperger syndrome: a guide for practitioners and carers. Chichester, UK: John Wiley \& Sons; 1998

6. Rescorla L. Cluster analytic identification of autistic preschoolers. J Autism Dev Disord 1988; 18: 475-92.

7. Achenbach TM, Edelbrock CS. Manual for the Child Behavior Checklist and Revised Child Behavior Profile. Burlington, TV: University Associates in Psychiatry; 1985.

8. American Psychiatric Association. Diagnostic and statistical manual of mental disorders, third edition (DSM-III). Washington DC: APA; 1980.

9. American Psychiatric Association. Diagnostic and statistical manual of mental disorders, fourth edition (DSM-IV). Washington DC: APA; 1994.

10. American Psychiatric Association. Diagnostic and statistical manual of mental disorders, fourth edition revised (DSM-IV-TR). Washington DC: APA; 2001.

11. Wood JJ, Gadow, KD. Exploring the nature and function of anxiety in youth with autism spectrum disorders Clin Psychol Sci Pract 2010; 17: 281-92.

12. Paula I. Diagnóstico diferencial entre el trastorno obsesivo compulsivo y los patrones de comportamiento, actividades e intereses restringidos y repetitivos en los trastornos del espectro autista. Revista de Psiquiatría y Salud Mental 2012 http://dx.doi.org/10.1016/j.rpsm.2012.07.005.

13. Van Steensel FJA, Bögels SM, Perrin S. Anxiety disorders in children and adolescents with autistic spectrum disorders: a meta-analysis. Clin Child Fam Psychol Rev 2011; 14: 302-17.

14. White SW, Oswald D, Ollendick T, Scahill L. Anxiety in children and adolescents with autism spectrum disorders. Clin Psychol Rev 2009; 29: 216-29.

15. MacNeil BM, Lopes VA, Minnes PM. Anxiety in children and adolescents with autism spectrum disorder. Research in Autism Spectrum Disorders 2009; 3: 1-21.

16. Simonoff E, Pickles A, Charman T, Chandler S, Loucas T, Baird G. Psychiatric disorders in children with autism spectrum disorders: prevalence, comorbidity, and associated factors in a population-derived sample. J Am Acad Child Adolesc Psychiatry 2008; 47: 921-9.

17. De Bruin EI, Ferdinand RF, Meester S, De Nijs PF, Verheij F.
High rates of psychiatric co-morbidity in PDD-NOS. J Autism Dev Disord 2007; 37: 877-86.

18. Clark DA, Beck AT. Terapia cognitiva para trastornos de ansiedad. Serie Psicoterapias Cognitivas. Biblioteca de Psicología. Bilbao: Desclée de Brouwer; 2012.

19. Hartley CA, Phelps EA. Anxiety and decision-making. Biol Psychiatry 2012; 72: 113-8.

20. Groden J, Baron MG, Groden G. Assessment and coping strategies. In Baron MG, Groden J, Groden G, Lipsitt LP, eds. Stress and coping in autism. New York: Oxford University Press; 2006.

21. Portway SM, Johnson B. Do you know I have Asperser's syndrome? Risks of a non-obvious disability. Health Risk Soc 2005; 7: 73-83.

22. White SW, Roberson-Nay R. Anxiety, social deficits, and loneliness in youth with autism spectrum disorders. J Autism Dev Disord 2009; 39: 1006-13.

23. Bauminger N, Schulman C, Agam G. Peer interaction and loneliness in high-functioning children with autism. J Autism Dev Disord 2003; 33: 489-507.

24. Sukhodolsky DG, Scahill L, Gadow KD, Arnold LE, Aman MG, McDougle CJ, et al. Parent-rated anxiety symptoms in children with pervasive developmental disorders: frequency and association with core autism symptoms and cognitive functioning. J Abnorm Child Psychol 2008; 36: 117-28.

25. Tantam $D$. The challenge of adolescents and adults with Asperger syndrome. Child Adolesc Psychiatr Clin N Am 2003; $12: 143-63$

26. Rodgers J, Glod M, Connolly B, McConachie H. The relationship between anxiety and repetitive behaviours in autism spectrum disorder. J Autism Dev Disord 2012; doi 10.1007/s10803-0121531.

27. Ooi YP, Lam CM, Sung M, Tan WTS, Goh TJ, Fung DSS, et al. Effects of cognitive-behavioural therapy on anxiety for children with high-functioning autistic spectrum disorders. Singapore Med J 2008; 49: 215-20.

28. Spiker MA, Lin CE, Van Dyke M, Wood JJ. Restricted interests and anxiety in children with autism. Autism 2011; 16: 306-20.

29. Joosten AV, Bundy AC, Einfeld SL. Intrinsic and extrinsic motivation for stereotypic and repetitive behavior. J Autism Dev Disord 2009; 39, 521-31.

30. Kashdan TB, McKnight PE, Richey JA, Hofmann SG. When social anxiety disorder co-exists with risk-prone, approach behavior: investigating a neglected, meaningful subset of people in the National Comorbidity Survey-Replication. Behav Res Ther 2009; 47: 559-68.

31. Leary MR, Twenge JM, Quinlivan E. Interpersonal rejection as a determinant of anger and aggression. Pers Social Psychol Rev 2006; 10: 111-32.

32. DeWall CN, Buckner JD, Lambert NM, Cohen AS, Fincham FD. Bracing for the worst, but behaving the best: social anxiety, hostility, and behavioral aggression. J Anxiety Disord 2010; 24: 260-8.

33. Reijntjes A, Thomaes S, Kamphuis JH, Bushman BJ, De Castro BO, Telch MJ. Explaining the paradoxical rejection-aggression link: the mediating effects of hostile intent attributions, anger, and decreases in state self-steem on peer rejection-induced aggression in youth. Pers Soc Psychol B 2011; 37: 955-63.

34. Research Units on Pediatric Psychopharmacology Autism Network. Risperidone in children with autism and serious behavioral problems. N Engl J Med 2002; 347: 314-21.

35. Farmer CA, Aman MG. Aggressive behavior in a sample of children with autism spectrum disorders. Research in Autism Spectrum Disorders 2011; 5: 317-23.

36. White SW, Kreiser NL, Pugliese C, Scarpa A. Social anxiety mediates the effect of autism spectrum disorder characteristics on hostility in young adults. Autism 2012; 16: 453-64.

37. Verte S, Geurts HM, Roeyers H, Oosterlaan J, Sergeant JA. Executive functioning in children with an autism spectrum disorder: can we differentiate within the spectrum? J Autism Dev Disord 2006; 36: 351-72.

38. Sofronoff K, Attwood T, Hinton S, Levin I. A randomized 
controlled trial of a cognitive behavioural intervention for anger management in children diagnosed with Asperger syndrome. J Autism Dev Disord 2007; 37: 1203-14.

39. Laurent AC, Rubin E. Emotional regulation challenges in Asperger syndrome and high functioning autism. Top Lang Disord 2004; 24: 4.

40. Williamson S, Craig J, Slinger R. Exploring the relationship between measures of self-esteem and psychological adjustment among adolescents with Asperger syndrome. Autism 2008; 12: 391-402.

41. Kuusikko S, Pollock-Wurman R, Jussila K, Carter AS, Mattila ML, Ebeling $\mathrm{H}$, et al. Social anxiety in high-functioning children and adolescents with autism and Asperger syndrome. J Autism Dev Disord 2008; 38: 1697-709.

42. Van Roekel E, Scholte R, Didden R. Bullying among adolescents with autism spectrum disorders: prevalence and perception. J Autism Dev Disord 2010; 40: 63-73.

43. Hurtig T, Kuusikko S, Matilla ML, Haapsamo H, Ebeling H, Jussila K, et al. Multi-informant reports of psychiatric symptoms among high-functioning adolescents with Asperger syndrome or autism. Autism 2009; 13: 583-98.

44. Lopata C, Toomey JA, Fox JD, Volker MA, Chow SY, Thomeer ML, et al. Anxiety and depression in children with HFASDs: symptom levels and source differences. J Abnorm Child Psychol 2010; 38: 765-76.

45. Mazefsky CA, Kao J, Oswald DP. Preliminary evidence suggesting caution in the use of psychiatric self-report measures with adolescents with high-functioning autism spectrum disorders. Research in Autism Spectrum Disorders 2011; 5: 164-74.

46. Farrugia S, Hudson J. Anxiety in adolescents with Asperger syndrome: negative thoughts, behavioral problems, and life interference. Focus Autism Other Dev Disabil 2006; 21: 25-35.

47. Russell E, Sofronoff K. Anxiety and social worries in children with Asperger syndrome. Aust N Z J Psychiatry 2005; 39: 633-8.

48. Achenbach TM, McConaughy SH, Howell CT. Child/ adolescent behavioral and emotional problems: implications of cross-informant correlations for situational specificity. Psychol Bull 1987; 101: 213-32.

49. Reynolds CR, Kamphaus RW. Behavior assessment system for children. Circle Pines: AGS; 2004.

50. Gadow KD, Roohi J, DeVincent CJ, Kirsch S, Hatchwell E. Association of COMT (Val158Met) and BDNF (Val66Met) gene polymorphisms with anxiety, ADHD and tics in children with autism spectrum disorder. J Autism Dev Disord 2009; 39: 1542-51

51. Weisbrot DM, Gadow KD, DeVicent CJ, Pomeroy J. The presentation of anxiety in children with pervasive developmental disorders. J Child Adolesc Psychopharmacol 2005; 15: 477-96.

52. Kim MJ, Gee DG, Loucks RA, Davis FC, Whalen PJ. Anxiety dissociates dorsal and ventral medial prefrontal cortex functional connectivity with the amygdala at rest. Cereb Cortex 2010; 21: 1667-73

53. Mineka S, Zinbarg R. A contemporary learning theory perspective on the etiology of anxiety disorders. It's not what you thought it was. Am Psychol 2006; 61: 10-26.

54. Kim MJ, Loucks RA, Palmer AL, Brown AC, Solomon KM, Marchante AN, et al. The structural and functional connectivity of the amygdala: from normal emotion to pathological anxiety. Behav Brain Res 2011; 223: 403-10.

55. Bishop SJ. Neurocognitive mechanisms of anxiety: an integrative account. Trends Cogn Sci 2007; 11: 307-16.

56. Ochsner NK, Gross JJ. The cognitive control of emotion. Trends Cogn Sci 2005; 9: 242-9.

57. Davis $\mathrm{M}$, Whalen PJ. The amygdala: vigilance and emotion. Mol Psychiatry 2001; 6: 13-34.

58. LeDoux JE. The amygdala. Curr Biol 2007; 17: 868-74

59. Phillips ML, Drevets WC, Rauch SL, Lane R. Neurobiology of emotion perception I: the neural basis of normal emotion perception. Biol Psychiatry 2003; 54: 504-514.

60. Etking A, Klemenhagen KC, Dudman JT, Rogan MT, Hen R, Kandel ER, et al. Individual differences in trait anxiety predict the response of the basolateral amygdala to unconsciously processed fearful faces. Neuron 2004; 44: 1043-55.
61. Stein MB, Simmons AN, Feinstein JS, Paulus MP. Increased amygdala and insula activation during emotion processing in anxiety-prone subjects. Am J Psychiatry 2007; 164: 318-27.

62. Lissek S, Powers AS, McClure EB, Phelps EA, Woldehawariat G, Grillon C, et al. Classical fear conditioning in the anxiety disorders: a meta-analysis. Behav Res Ther 2005; 43: 1391-424.

63. Indovina I, Robbins T, Núñez-Elizande A, Dunn B, Bishop SJ. Fear conditioning mechanism associated with trait vulnerability to anxiety. Neuron 2011; 69: 563-71.

64. Somerville LH, Kim H, Johnstone T, Alexander AL, Whalen PJ. Human amygdala responses during presentation of happy and neutral faces: correlations with state anxiety. Biol Psychiatry 2004; 55: 897-903.

65. Fanselow MS. Contextual fear, gestalt memories, and the hippocampus. Behav Brain Res 2000; 110: 73-81.

66. Bannerman DM, Rawlins JN, McHugh SB, Deacon RM, Yee BK, Bast $\mathrm{T}$, et al. Regional dissociations within the hippocampus -memory and anxiety. Neurosci Biobehav Rev 2004; 28: 273-83.

67. Richardson MP, Strange BA, Dolan RJ. Encoding of emotional memories depends on amygdala and hippocampus and their interactions. Nat Neurosci 2004; 7: 278-85.

68. Lissek S, Rabin S, Heller RE, Lukengbaugh D, Geraci M, Pine DS, et al. Overgeneralitzation of conditioned fear as a pathogenic marker of panic disorder. Am J Psychiatry 2010; 167: 47-55.

69. Pezawas L, Meyer-Lindenberg A, Drabant EM, Verchinski BA, Muñoz KE, Kolachana BS, et al. 5-HTTLPR polymorphism impacts human cingulate-amygdala interactions: a genetic susceptibility mechanism for depression. Nat Neurosci 2005; 8: 828-34.

70. Kim MJ, Whalen PJ. The structural integrity of an amygdalaprefrontal pathway predicts trait anxiety. J Neurosci 2009; 29: 11614-8.

71. Amaral DG, Bauman MD, Schumann CM. The amygdale and autism: implications from non-human primate studies. Gene Brain Behav 2003; 2: 295-302.

72. Amaral DG, Corbett BA. The amygdala, autism and anxiety. Novartis Foundation Symposium 2003; 251: 177-87.

73. Baron-Cohen S, Ring HA, Bullmore ET, Wheelwright S, Ashwin C, Williams SC. The amygdala theory of autism. Neurosci Biobehav Rev 2000; 24: 355-64.

74. Salmond, CH, Ashburner J, Connelly A, Friston KJ, Gadian DG. The role of the medial temporal lobe in autistic spectrum disorders. Eur J Neurosci 2005; 22: 764-72.

75. Schultz RT. Developmental deficits in social perception in autism: the role of the amygdala and fusiform face area. Int J Dev Neurosci 2005; 23: 125-41.

76. Bauman M, Kemper TL. Histoanatomic observations of the brain in early infantile autism. Neurology 1985; 35: 866-74.

77. Sparks BF, Friedman SD, Shaw DW, Aylward E, Echelard D, Artru AA, et al. Brain structural abnormalities in young children with autism spectrum disorder. Neurology 2002; 59: 184-92.

78. Mosconi MW, Cody-Hazlett H, Poe MD, Gerig G, GimpelSmith R, Piven J. Longitudinal study of amygdala volume and joint attention in 2- to 4-year-old children with autism. Arch Gen Psychiatry 2009; 66: 509-16.

79. Schumann CM, Barnes CC, Lord C, Courchesne E. Amygdala enlargement in toddlers with autism related to severity of social and communication impairments. Biol Psychiatry 2009; 66: 942-9.

80. Groen W, Teluij M, Buitelaar J, Tendolkar I. Amygdala and hippocampus enlargement during adolescence in autism. J Am Acad Child Adolesc Psychiatry 2010; 49: 552-60.

81. Rojas DC, Smith JA, Benkers TL, Camou SL, Reite ML, Rogers SJ. Hippocampus and amygdala volumes in parents of children with autistic disorder. Am J Psychiatry 2004; 161: 2038-44.

82. Rojas DC, Peterson E, Winterrowd E, Reite ML, Rogers SJ, Tregellas JR. Regional gray matter volumetric changes in autism associated with social and repetitive behavior symptoms. BMC Psychiatry 2006; 6: 56.

83. Schumann CM, Hamstra J, Goodlin-Jones BL, Lotspeich LJ, 
Kwon $\mathrm{H}$, Buonocore $\mathrm{MH}$, et al. The amygdala in enlarged in children but not adolescents with autism; the hippocampus in enlarged at all ages. J Neurosci 2004; 24: 6392-401.

84. Clodagh M, Murphy Q, Deeley EM, Daly C, Ecker FM, O'Brien B, et al. Anatomy and aging of the amygdala and hippocampus in autism spectrum disorder: an in vivo magnetic resonance imaging study of Asperger syndrome. Autism Res 2011; 4: 1-10.

85. Howard MA, Cowell PE, Boucher J, Broks P, Mayes A, Farrant A, et al. Convergent neuroanatomical and behavioural evidence of an amygdala hypothesis of autism. Neuroreport 2000; 11: 2931-5.

86. Bigler ED, Tate DF, Neeley ES, Wolfson LJ, Miller MJ, Rice SA, et al. Temporal lobe, autism, and macrocephaly. AJNR Am J Neuroradiol 2003; 24: 2066-76.

87. Haznedar MM, Buchsbaum MS, Wei TC, Hof PR, Cartwright C, Bienstock CA, et al. Limbic circuitry in patients with autism spectrum disorders studied with positron emission tomography and magnetic resonance imaging. Am J Psychiatry 2000; 157: 1994-2001.

88. Palmen SJ, Durston S, Nederveen H, Van Engeland H. No evidence for preferential involvement of medial temporal lobe structures in high-functioning autism. Psychol Med 2006; 36: 827-34.

89. Dalton KM, Nacewicz BM, Alexander AL, Davidson RJ. Gaze-fixation, brain activation, and amygdala volume in unaffected siblings of individuals with autism. Biol Psychiatry 2007; 61: 512-20.

90. Aylward EH, Minshew NJ, Goldstein G, Honeycutt NA, Augustine AM, Yates KO, et al. MRI volumes of amygdala and hippocampus in non-mentally retarded autistic adolescents and adults. Neurology 1999; 53: 2145-50.

91. Pierce K, Muller RA, Ambrose J, Allen G, Courchesne E. Face processing occurs outside the fusiform 'face area' in autism: evidence from functional MRI. Brain 2001; 124: 2059-73.

92. Ecker C, Marquand A, Mourao-Miranda J, Johnston P, Daly EM, Brammer MJ, et al. Describing the brain in autism in five dimensions -magnetic resonance imaging-assisted diagnosis of autism spectrum disorder using a multiparameter classification approach. J Neurosci 2010; 30: 10612-23.

93. Palmen SJ, Van Engeland H, Hof PR, Schmitz C. Neuropathological findings in autism. Brain 2004; 127: 2572-83.

94. Schumann CM, Amaral DG. Stereological analysis of amygdala neuron number in autism. J Neurosci 2006; 26: 7674-9.

95. Schumann CM, Amaral DG. The human amygdala in autism New York: Guilford Press; 2009.

96. Raymond GV, Bauman ML, Kemper TL. Hippocampus in autism: a Golgi analysis. Acta Neuropathol 1996; 91: 117-9.

97. Adolphs R. The social brain: neural basis of social knowledge. Annu Rev Psychol 2009; 60: 693-716.

98. Corbett BA, Carmean V, Ravizza S, Wendelken C, Henry ML, Carter C, et al. A functional and structural study of emotion and face processing in children with autism. Psychiatry Res 2009; 173: 196-205.

99. Dziobek I, Bahnemann M, Convit A, Heekeren HR. The role of the fusiform-amygdala system in the pathophysiology of autism. Arch Gen Psychiatry 2010; 67: 397-405.

100. Monk CS, Weng SJ, Wiggins JL, Kurapati N, Louro HM Carrasco M, et al. Neural circuitry of emotional face processing in autism spectrum disorders. J Psychiatry Neurosci 2010; 35: 105-14.

101. Schumann CM, Bauman MD, Amaral DG. Abnormal structure or function of the amygdala is a common component of neurodevelopmental disorders. Neuropsychology 2011; 49: 745-59.

102. Nordahl CW, Scholz R, Yang X, Buonocore MH, Simon T, Rogers $S$, et al. Increased rate of amygdala growth in children aged 2 to 4 years with autism spectrum disorders: a longitudinal study. Arch Gen Psychiatry 2010; 69: 53-61.

103. Roozendaal B, McEwen BS, Chattarji S. Stress, memory and amygdala. Nature 2009; 10: 423-33.

104. Hill SY, Tessner K, Wang S, Carter H, McDermott M.
Temperament at 5 years of age predicts amygdala and orbitofrontal volume in the right hemisphere in adolescence. Psychiatry Res 2010; 182: 14-21.

105. Beesdo K, Lau JY, Guyer AE, McClure-Tone EB, Monk CS, Nelson EE, et al. Common and distinct amygdala-function perturbations in depressed vs anxious adolescents. Arch Gen Psychiatry 2009; 66: 275-85.

106. Guyer AE, Lau JY, McClure-Tone EB, Parrish J, Shiffrin ND, Reynolds RC, et al. Amygdala and ventrolateral prefrontal cortex function during anticipated peer evaluation in pediatric social anxiety. Arch Gen Psychiatry 2008; 65: 1303-12.

107. McClure EB, Adler A, Monk CS, Cameron J, Smith S, Nelson EE, et al. fMRI predictors of treatment outcome in pediatric anxiety disorders. Psychopharmacology (Berl) 2007; 191: 97-105.

108. Weng S, Wiggins J, Peltier S, Carrasco M, Risi S, Lord C, et al. Alterations of resting state functional connectivity in the default network in adolescents with autism spectrum disorders. Brain Res 2010; 13: 202-14

109. McEwen BS. Physiology and neurobiology of stress and adaptation: central role of the brain. Physiol Rev 2007; 87: 873-904.

110. Juranek J, Filipek PA, Berenji GR, Modahl C, Ossan K, Spence MA. Association between amygdala volume and anxiety level: magnetic resonance imaging (MRI) study in autistic children. J Child Neurol 2006; 21: 1051-8.

111. Munson J, Dawson G, Abbott R, Faja S, Webb SJ, Friedman SD, et al. Amygdalar volume and behavioral development in autism. Arch Gen Psychiatry 2006; 63: 686-93.

112. Van Eijndhoven P, Van Wingen G, Van Oijen K, Rijpkema M, Goraj B, Jan-Verkes R, et al. Amygdala volume marks the acute state in the early course of depression. Biol Psychiatry 2009; 65: 812-9.

113. Frodl T, Moller HJ, Meisenzahl E. Neuroimaging genetics: new perspectives in research on major depression? Acta Psychiatr Scand 2008; 118: 363-72.

114. Chattarji S, Vyas A, Mitra R, Rao BS. Effects of chronic unpredictable and immobilization stress on neuronal plasticity in the rat amygdala and hippocampus. Soc Neurosci Abs 2000; $26: 1533$

115. Sheline YI, Wang PW, Gado MH, Csernanski JG, Vannier MW. Hippocampal atrophy in recurrent major depression. Proc Natl Acad Sci U S A 1996; 93: 3908-13.

116. Sheline YI, Gado MH, Price JL. Amygdala core nuclei volumes are decreased in recurrent major depression. Neuroreport 1998; 9: 2023-8.

117. McEwen BS. Stress and hippocampal plasticity. Annu Rev Neurosci 1999; 22: 105-22.

118. Erath SA, Flanagan KS, Bierman KL. Social anxiety and peer relations in early adolescence: behavioral and cognitive factors, J Abnorm Child Psychol 2007; 35: 405-16.

119. Rao PA, Beidel DC, Turner SM, Ammerman RT, Crosby LE, Sallee FR. Social anxiety disorder in childhood and adolescence: descriptive psychopathology. Behav Res Ther 2007; 45: 1181-91.

120 . Wood JJ. Effect of anxiety reduction on children's school performance and social adjustment. Dev Psychol 2006; 42: 345-9.

121. Reaven JA. The treatment of anxiety symptoms in youth with high functioning autism spectrum disorder: developmental considerations for parents. Brain Res 2011; 1380: 255-63.

122. Brown TA, Campbell LA, Lehman CL, Grisham JR, Mancill RB. Current and lifetime comorbidity of the DSM-IV anxiety and mood disorders in a large clinical simple. J Abnorm Psychol 2001; 110: 585-99.

123. Lipsky D. From anxiety to meltdown. London: Jessica Kingsley; 2009.

124. Billstedt E, Gillberg IC, Gillberg C. Autism in adults: symptom patterns and early child predictors. Use of the DISCO in a community sample followed from childhood. J Child Psychol Psychiatry 2007; 48: 1102-10.

125. Chalfant AM, Rapee R, Carroll L. Treating anxiety disorders in children with high-functioning autism spectrum disorders: a controlled trial. J autism Dev Disord 2007; 37: 1842-57. 
126. Reaven J, Blakeley-Smith A, Culhane-Shelburne K, Hepburn S. Group cognitive behavior therapy for children with highfunctioning autism spectrum disorders and anxiety: a randomized trial. J Child Psychol Pychiatry 2012; 53: 410-9.
127. Wood JJ, Drahota A, Sze K, Har K, Chiu A, Langer D. Cognitive behavioral therapy for anxiety in children with autism spectrum disorders: a randomized, controlled trial. J Child Psychol Psychiatry 2009; 50: 224-34.

\section{Co-occurrence of anxiety and autism. The social error and allostatic load hypotheses}

Introduction. The concept of comorbidity in neurodevelopmental disorders like autism is sometimes ambiguous. The cooccurrence of anxiety and autism is clinically significant, yet it is not always easy to determine whether it is a 'real' comorbidity, where the two comorbid conditions are phenotypically and aetiologically identical to what that anxiety would mean in persons with a neurotypical development, whether it is an anxiety that has been phenotypically modified by the pathological processes of the autism spectrum disorders, thus resulting in a specific variant of these latter, or whether we are dealing with a false comorbidity resulting from rather inaccurate differential diagnoses.

Development. The article puts forward two hypotheses to explain this co-occurrence, which provide each other with feedback and are little more than our reflections on the scientific evidence we have available today, but expressed aloud. The first is the 'social error' hypothesis, which considers that the maladjustments in the social behaviour of persons with autism (which arises from alterations affecting the processes involved in social cognition) help to aggravate anxiety in autism. The second hypothesis, referring to allostatic load, holds that anxiety is a response to chronic stress, wear or exhaustion that is produced by the hyperactivation of certain structures in the limbic system.

Conclusions. The prototypical manifestations of anxiety present in the person with autism are not always related with the same biopsychosocial variables as those observed in persons without autism. Evidence points to hyper-reactive flee-or-fight responses (hypervigilance) when the person finds him or herself outside their comfort zone, and supports the hypotheses of 'social error' and of decompensation of the allostatic mechanism that makes it possible to cope with stress.

Key words. Allostatic load. Anxiety disorders. Autistic spectrum disorders. Comorbidity. Co-occurrence. 